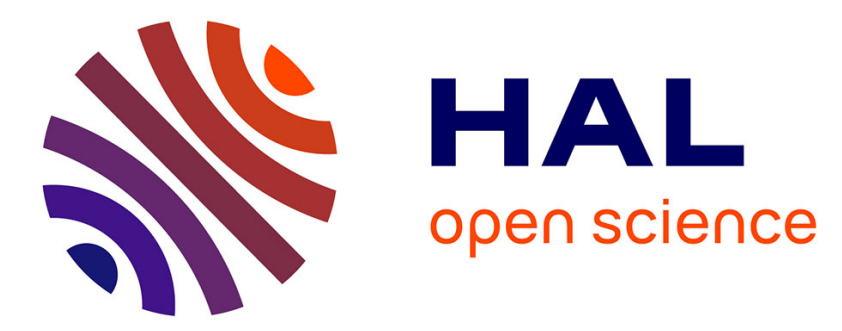

\title{
Linking growth mode to lengths of single-walled carbon nanotubes
}

Maoshuai He, Yann Magnin, Hakim Amara, Hua Jiang, Hongzhi Cui, Frederic

Fossard, Alice Castan, Esko Kauppinen, Annick Loiseau, Christophe Bichara

\section{- To cite this version:}

Maoshuai He, Yann Magnin, Hakim Amara, Hua Jiang, Hongzhi Cui, et al.. Linking growth mode to lengths of single-walled carbon nanotubes. Carbon, 2017, 113, pp.231-236. 10.1016/j.carbon.2016.11.057 . hal-01432079

\section{HAL Id: hal-01432079 \\ https://hal.science/hal-01432079}

Submitted on 2 Jul 2021

HAL is a multi-disciplinary open access archive for the deposit and dissemination of scientific research documents, whether they are published or not. The documents may come from teaching and research institutions in France or abroad, or from public or private research centers.
L'archive ouverte pluridisciplinaire HAL, est destinée au dépôt et à la diffusion de documents scientifiques de niveau recherche, publiés ou non, émanant des établissements d'enseignement et de recherche français ou étrangers, des laboratoires publics ou privés.

\section{(c)(1)}

Distributed under a Creative Commons Attribution| 4.0 International License 


\section{Linking Growth Mode to Lengths of Single-Walled Carbon Nanotubes}

Maoshuai He, ${ }^{1,2 *}$ Yann Magnin, ${ }^{3}$ Hakim Amara, ${ }^{2}$ Hua Jiang, ${ }^{4}$ Hongzhi Cui, ${ }^{1 *}$ Frédéric Fossard, ${ }^{2}$ Alice Castan, ${ }^{2}$ Esko Kauppinen, ${ }^{4}$ Annick Loiseau, ${ }^{2}$ Christophe Bichara ${ }^{3}$

1. School of Materials Science and Engineering, Shandong University of Science and Technology, 266590 Qingdao, People's Republic of China

2. Laboratoire d'Étude des Microstructures, ONERA-CNRS, BP 72, 92322 Châtillon CEDEX, France

3. Aix-Marseille University and CNRS, CINaM UMR 7325, 13288 Marseille, France

4. Department of Applied Physics, Aalto University School of Science, P.O. Box 15100, FI-00076 Aalto, Finland.

\section{Corresponding Author}

* Correspondence should be addressed to: Maoshuai He, Email: maoshuai.he@sdust.edu.cn; Hongzhi Cui, Email: cuihongzhi1965@163.com 


\begin{abstract}
Elucidating the key factors that determine the lengths of single-walled carbon nanotubes (SWCNTs) is of great importance for understanding the origin of chiral selectivity. We use transmission electron microscopy to thoroughly investigate as-grown SWCNTs. The lengths and growth modes of SWCNTs were decided by bright-field imaging. Their respective chiral angles were calculated on the basis of nanobeam diffraction patterns. Systematic investigations reveal that there is no correlation between the SWCNT length and its chiral angle. Instead, it shows that SWCNT lengths depend more on their growth mode, i.e. the link between SWCNT and its seeding catalyst particle. Atomistic computer simulations demonstrate that low carbon fractions in the catalyst lead to so-called tangential growth, with a partial wetting of the metal in the tube, where metal catalyst tends to be deactivated by graphite layer encapsulation and results in short SWCNTs. In contrast, a high carbon concentration inside metal particle favors perpendicular growth modes, where only the tube lip is in contact with the catalyst. Catalysts adopting perpendicular mode could have a longer lifetime, thus catalyze the growth of long SWCNTs. Finally, the carbon concentration related growth mode was applied to interpret diverse SWCNT growth results.
\end{abstract}

Keywords: single-walled carbon nanotube, growth kinetics, growth mode, SWCNT length, chirality selectivity

\title{
Introduction
}

Searching for chiral selectivity during the chemical vapor deposition (CVD) growth of single-walled carbon nanotubes (SWCNTs) is a much coveted goal that has been partially reached in a number of experiments [1-6]. However, the rationale behind these successful achievements has 
never been fully understood, precluding coherent synthesis strategies. So far, two theories have been proposed to interpret the chirality selectivity: one is nucleation control, where SWCNTs with specific chirality nucleate on the catalyst nanoparticles $[2,3,5]$, accounting for the controlled growth. The other is SWCNT growth kinetic control [7-9], where SWCNTs with certain structure grow faster and longer than others, leading to their selective growth characterization results. In this context, SWCNTs length, diameter and chirality together with the size of the nanoparticle from which they grow appear as important quantities to investigate, in order to gain a better understanding of SWCNT growth mechanisms.

In order to clarify possible factors that affect SWCNT lengths, a prominent screw-dislocation model has been proposed [7], with the main result that the nanotube growth rate should be proportional to its chiral angle. This model was extended by further taking the nanotube/catalyst interface thermodynamics into account [8]. Based on this approach, SWCNT growth rate and possibly length should be correlated with its chiral angle. However, the finding of a link between SWCNT growth rate/length and chiral angle is at odds with the overall conclusions of some studies [10, 11]. Using in situ Raman spectroscopy, Rao et al.[10] measured the growth rates of nine individual tubes and found them proportional to their chiral angles, in agreement with the above model [7]. However, recent Raman mapping on SWCNTs with five kinds of chiralities showed no length dependence on tube chiral angle [11].

The discrepancy in experimental findings is likely due to the limited amount of data acquired in the above experiments $[10,11]$. In both cases, Raman spectroscopy is used to follow isolated SWCNTs grown on surfaces. As the resonance window of Raman scattering for a 
bare-isolated SWCNT is very narrow $(\sim 10 \mathrm{meV})[12,13]$, for a given laser wavelength, the probability to satisfy the resonant condition for observing an individual SWCNT is less than $1 \%$. Moreover, the relatively low spatial resolution of Raman spectroscopy, which is limited by diffraction to about half the wavelength of the excitation light, challenges researchers to measure tubes which are not very long [11]. Consequently, in order to precisely measure tube lengths and explore the factors that could affect them, we should resort to other more reliable techniques.

In this work, we combine high-resolution transmission electron microscopy (TEM) and nanobeam electron diffraction (ED) to thoroughly characterize SWCNTs synthesized on porous $\mathrm{SiO}_{2}$ membrane. We stress that the strength of our approach is first, to measure, on a given tube, its chiral angle, length and the nature of its link to seed particle from which it has grown and second, to perform these measurements in a statistical way, independently of the nature and morphology of the tubes inspected. First of all, the tube length and chiral angle were determined, with the result that we found no correlation between the SWCNT length and its chiral angle. Secondly, we demonstrated the SWCNT length could be related with the tube growth mode, depending on links between tubes and their seeding particles. Finally, computer simulation was applied to show that catalyst particles growing SWCNTs by a tangential mode, tend to be deactivated by fully wetting the inner part of the tube, accounting for the shorter SWCNTs obtained in these CVD conditions.

\section{Materials and methods}

\subsection{Preparation of Fe catalyst}

Iron hydroxide nanoparticles were prepared by hydrolysis of $\mathrm{FeCl}_{3}$ [14]. In brief, $\mathrm{FeCl}_{3}$ with a mass of $0.08 \mathrm{~g}$ was first dissolved in $5.0 \mathrm{~mL} \mathrm{H}_{2} \mathrm{O}$. The solution was added dropwise to $180 \mathrm{~mL}$ boiling 
water under stirring. The prepared Fe-containing colloid was diluted in ethanol and then dispersed onto TEM grid coated by porous silicon oxide layer. The dispersed Fe catalyst was calcined in air to remove possible contaminations.

\subsection{Growth of carbon nanotubes by CVD}

Growth of carbon nanotube was carried out in a horizontal CVD system equipped with a quartz tube (inner diameter: $40 \mathrm{~mm}$ ). The TEM grid with Fe nanoparticles was heated to $900{ }^{\circ} \mathrm{C}$ with a flow of He $(200 \mathrm{sccm})$. After reaching the desired temperature, $\mathrm{CH}_{4}$ with a flow rate of $200 \mathrm{sccm}$ was introduced into the reactor to replace He. After reaction for $2 \mathrm{~min}, \mathrm{CH}_{4}$ was switched off and the system was cooled down under the protection of He.

\subsection{Characterizations of carbon nanotubes and catalyst particles by TEM}

The morphology and structure of both catalyst and carbon nanotubes were characterized by a JEOL-2200FS FEG TEM/STEM and JEOL aberration-corrected ARM 200 Cold-FEG microscope operated at $80 \mathrm{kV}$. The TEM micrographs were used to measure the lengths of SWCNTs. Structural assignments of the SWCNTs are done by analyzing their nanobeam electron diffraction patterns [15].

\subsection{Calculations of interactions between SWCNTs and metal nanoparticles}

To study the interaction of carbon with nickel at the atomic level, we have developed a tight-binding model based on a description of the local electronic density of states of each atom at the fourth moment level. This provides an efficient tool to calculate the energies of systems containing a few hundreds of $\mathrm{Ni}$ and $\mathrm{C}$ atoms $[16,17]$. The energetic model is then implemented in 
a Monte Carlo code using either a canonical or grand canonical algorithm with fixed volume, temperature, number of $\mathrm{Ni}$ atoms, and carbon chemical potential [18].

\section{Results and Discussion}

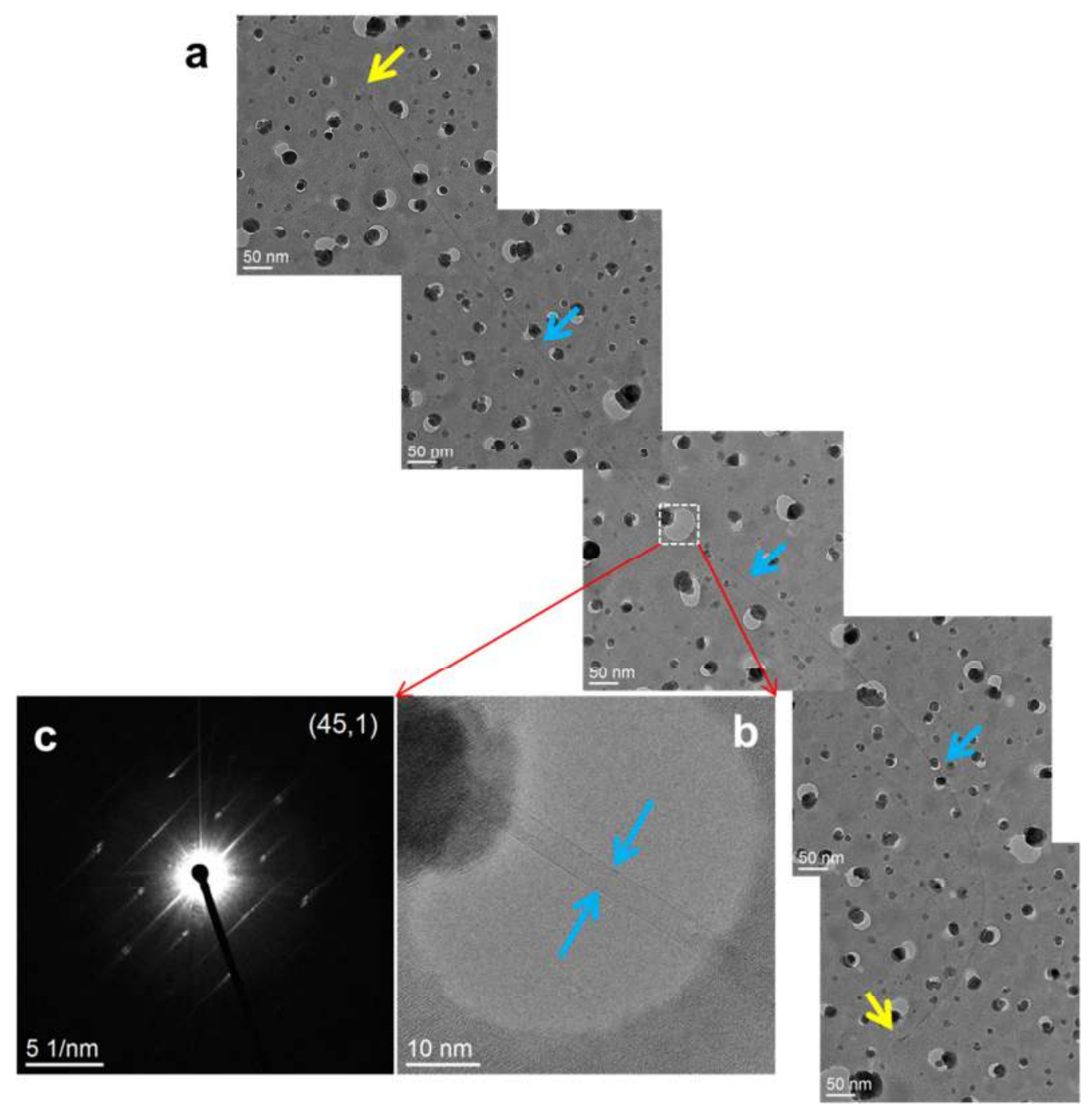

Figure 1. (a) TEM images of a $2100 \mathrm{~nm}$-long SWCNT (indicated by pacific light blue arrows), both ends are indicated by yellow arrows. (b) A high resolution TEM image of a SWCNT section crossing a hole. (c) Nanobeam electron diffraction pattern of the SWCNT.

Fig. 1a presents photograph of a $2.1 \mu \mathrm{m}$-long SWCNT measured by superimposing 5 TEM images taken along the tube. Both ends of are marked with yellow arrows. A high resolution image 
was taken from the SWCNT across a hole (Fig. 1b), with a measured diameter of $3.6 \mathrm{~nm}$. Nanobeam ED was performed on the suspended section of the SWCNT, the pattern of which is shown in Fig. 1c (a magnification is presented in ESI Fig. S1a). ED pattern of an SWCNT is consisted of many separated layer-lines parallel to each other. Based on the strategy of "intrinsic layer-line spacing" [15], the chirality indices of the SWCNT are assigned to $(45,1)$, with a chiral angle of $1.1^{\circ}$. Such an assignment is also confirmed by the simulated ED pattern of one $(45,1)$ SWCNT (ESI Fig. S1b). Fig. 2 depicts TEM micrograph of a short SWCNT (Fig. 2a) and its corresponding ED pattern (Fig. 2b). The tube has a length of only $40 \mathrm{~nm}$. Analyzing its ED pattern gives chiral indices of $(25,23)$, a tube with diameter of $3.3 \mathrm{~nm}$ and chiral angle of $28.6^{\circ}$. Such an assignment is also verified by the simulated ED pattern of a $(25,23)$ tube (Supporting Information Fig. S2) and the diameter of tube measured from Fig. 2a.

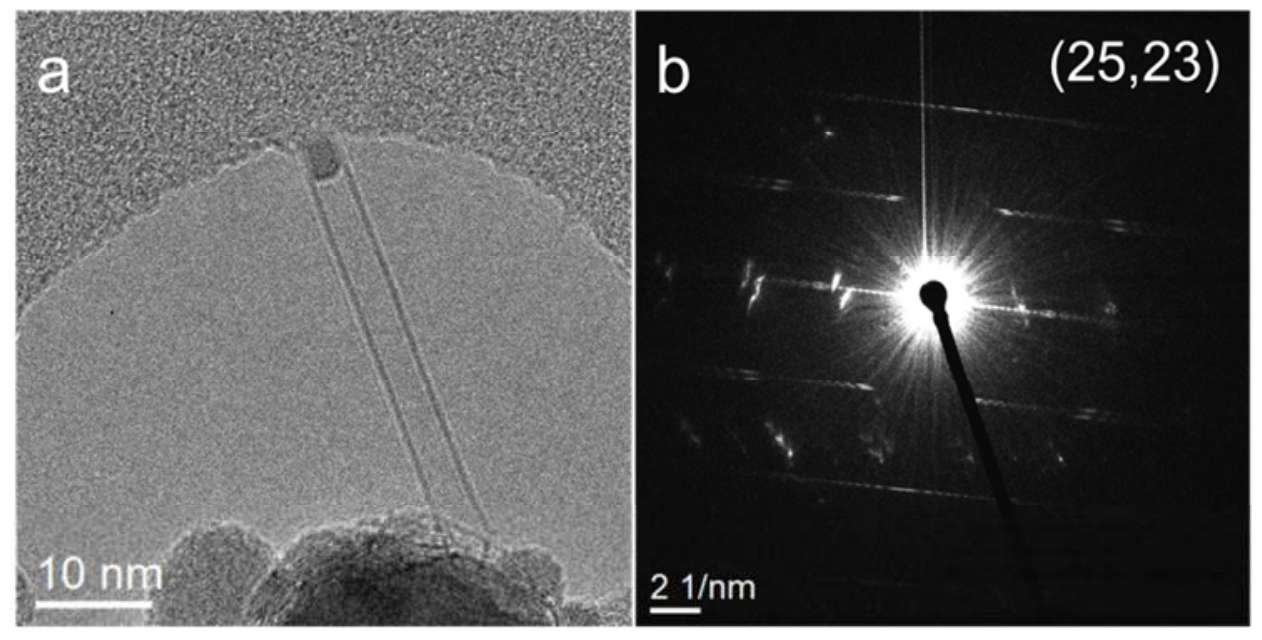

Figure 2. (a) TEM images of a 40-nm long SWCNT and (b) its nanobeam electron diffraction pattern. $(25,23)$ are the chiral indices deduced from the analysis of the diffraction pattern.

In contrast with Raman spectroscopy which can only detect SWCNTs that are in resonance with the laser wavelength, characterizations adopted here not only permit precise determination of 
SWCNT lengths by TEM, but facilitate unambiguous assignment of SWCNT chirality using nanobeam ED. Therefore, it allows investigating a number of randomly distributed SWCNTs with different chiralities, and very importantly without any preference. The diameter and chirality measurements were performed on 92 SWCNTs, grown in one experiment using $\mathrm{CH}_{4}$ as a feedstock. Fig. 3a depicts the plot of SWCNT lengths as a function of chiral angles. Obviously, no correlation could be identified between the length of the SWCNT and its chiral angle. For example, a $(25,13)$ SWCNT (ESI Fig. S3) with a chiral angle of $19.7^{\circ}$ has a length of only $140 \mathrm{~nm}$, while a $(27,14)$ one with similar chiral angle $\left(19.6^{\circ}\right)$ exhibits a length of more than $3200 \mathrm{~nm}$ (ESI Fig. S4). Similarly, the SWCNT length does not depend on the diameter of tube (ESI Fig. S5a) or the size of catalyst particle (ESI Fig. S5b).

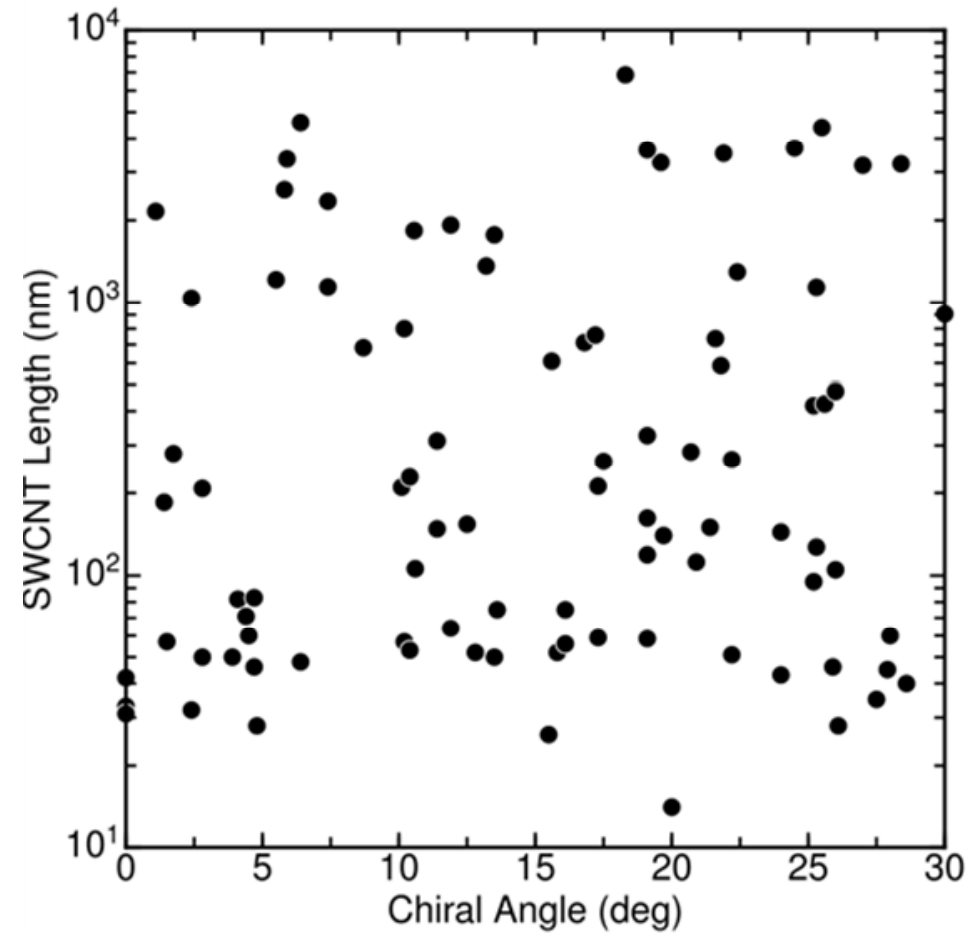

Figure 3. Lengths of SWCNTs synthesized by CVD using a Fe catalyst at $1173 \mathrm{~K}$ with $\mathrm{CH}_{4}$ as carbon feedstock, measured by TEM imaging, plotted as a function of their chiral angles. 
The conclusion is in agreement with the work of Inoue et al.[11], who reported that tube lengths show no clear dependence on chiral angles based on resonance Raman characterizations. However, due to the limitations of Raman spectroscopy, only 5 types of SWCNTs in the $2 \mathrm{n}+\mathrm{m}=$ 29 family were presented in their work [11]. Our work includes more SWCNTs, we can thus more safely conclude that the SWCNT lengths do not correlate with their chiral angles, which affects confidence in the general relevance of the proposed screw dislocation growth model [7], as far as the tube length reflects the growth rate.

Neglecting the role of the catalyst is clearly a rough simplification in the screw dislocation model and the role of the catalyst nanoparticle should be taken into account in more realistic models. Thus, further searching for correlations, we rely on previous work showing that the diameter ratio between SWCNT and its linked catalyst particle $\left(R_{d}=\mathrm{D}_{\mathrm{SWCNT}} / \mathrm{D}_{\mathrm{NP}}\right)$, was a relevant quantity to consider. It enabled classifying the SWCNT/nanoparticle contact into so-called "tangential", and "perpendicular" growth modes [19]. The stability of these different configurations could be related to the wetting properties of nanoparticles that are driven by their carbon dissolution abilities [20]. Recently, we revealed that the presence of carbon atoms dissolved inside the nanoparticle is required to nucleate and grow a tube [21]. This carbon content favors the dewetting of the nanoparticle with respect to the $\mathrm{sp}^{2}$ carbon wall, a necessary property to limit catalyst encapsulation and deactivation $[20,21]$. Therefore, we now focus on the relation between the diameters of the SWCNT and nanoparticle from which it grew, characterized by the diameter ratio $R_{d}$. 

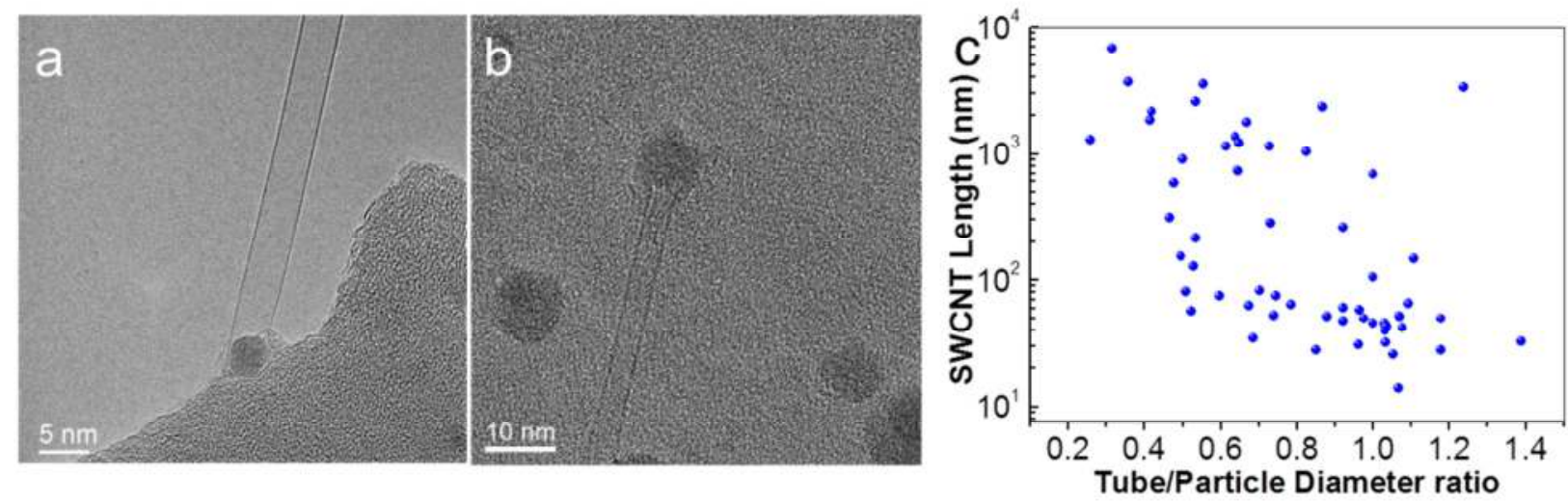

Figure 4. TEM images of SWCNTs grown by (a) tangential mode and (b) perpendicular mode from $\mathrm{CH}_{4}$ at $1173 \mathrm{~K}$ for $2 \mathrm{~min}$. (c) SWCNT length plots as a function of tube/particle diameter ratio.

Fig. 4a and 4b present TEM images of two SWCNTs and catalyst particles attached to them. As defined by Fiawoo et al. [19], SWCNT growth configurations in Fig. 4a has an $R_{d}$ of $\sim$, which can be identified as tangential mode. In contrast, SWCNT shown in Fig. 4b displays a perpendicular mode, with an $R_{d}$ of 0.44 . Fig. $4 \mathbf{c}$ presents the lengths of SWCNTs as a function of $R_{d}$. Bearing in mind that the logarithmic scale squeezes the one or two orders of magnitude difference between the lengths of the tubes we clearly see that tubes grown in perpendicular mode are much longer than those in tangential mode, with $R_{d} \approx 1$. Our TEM analysis thus clearly emphasizes a correlation between the length of SWCNTs and their growth modes, showing that perpendicular tubes grow longer and tangential ones grow shorter.

Computer simulations are applied to help figure out why tube length depends on tube growth mode. The nature of the metallic catalyst is assumed not to be essential, since both growth modes have been characterized for Fe and Co catalyst [19]. Consequently, the tight-binding model developed for the Ni-C system [16] was used to perform atomistic computer simulations. Such an interatomic energy model is implemented in a Monte Carlo code using (grand) canonical algorithms 
and has been applied to surface segregation of carbon and the catalytic nucleation/growth of SWCNTs [17, 20-22]. To study the differences in structure and stability of catalyst particles connected to SWCNTs in perpendicular and tangential modes at an atomic scale that cannot be accessed experimentally, we thus resort to these computer simulation tools.

We start by fixing long enough nanotubes with about 500 carbon atoms on Ni nanoparticles with 219 atoms. Two carbon fractions of $4 \%$ and $18 \%$ are chosen for the simulation. A full study of the effect of the fraction of carbon dissolved in the NP on the growth mode will be presented elsewhere. With fixed carbon fraction inside the nanoparticle, the system is relaxed to determine the equilibrium structures of Ni nanoparticles located at the tip of SWCNTs. Fig. 5a presents a visual inspection of the resulting structures. Nanoparticle with $4 \%$ carbon concentration tends to wet the inner part of the tube, in tangential mode geometry. A typical experimental evidence of such geometry is demonstrated in Fig. 5b. It is noted that although the configuration of metal particles shown here is a bit different from that presented in Fig. 4a, we assign both configurations as tangential mode [19]. As revealed by Diarra et al. [20], the morphology of a particle could change during SWCNT growth. The wetting tendency is more pronounced in the confined geometry of the tube, than on a flat graphene layer, where the contact angle was found to be around $80^{\circ}$ [23]. In the case of the largest possible carbon fraction inside $\mathrm{Ni}$ (18\%, Supporting Information Video 2), all Ni atoms stay outside and the tube wall becomes more perpendicularly oriented with respect to the surface of the nanoparticle. The results show that the equilibrium diameter ratio $R_{d}$ depends on the dissolved carbon atoms in particle: low carbon concentration in the nanoparticle leads to tangential growth with $R_{d} \approx 1$. In contrast, at the high carbon fraction, no Ni penetrates the tube cavity, and thus $R_{d}<1$, corresponding to the perpendicular mode situation. 

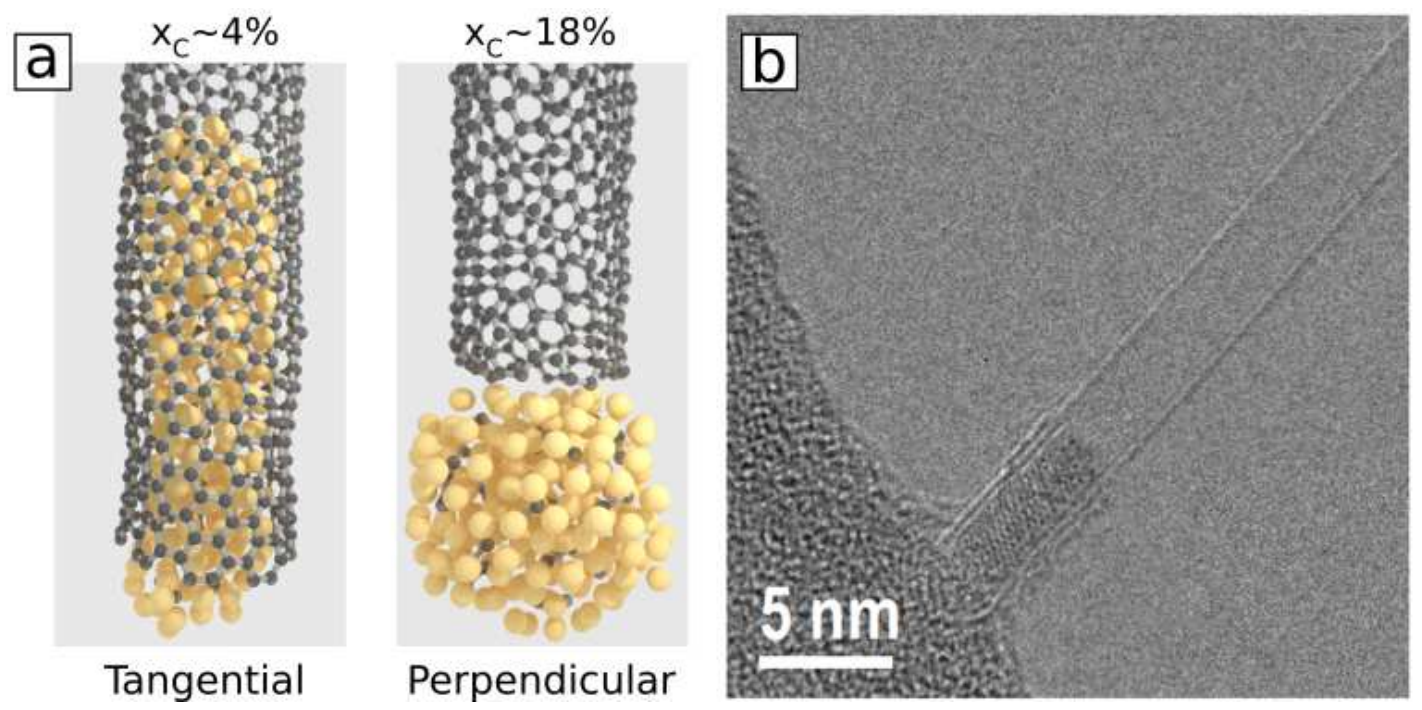

Figure 5. (a) Sketch of nanotube/nanoparticles geometries resulting from our Monte Carlo relaxations at $1200 \mathrm{~K}$, ranging from tangential (left) to perpendicular (right). (b) A TEM image of nanoparticle encapsulated in the tip of the SWCNT.

Besides determining tube growth mode, the carbon fraction inside liquid shell of catalyst particle [22] also affects the adhesion energy between carbon walls and the particle. As discussed by previous Monte Carlo calculations in which carbon atoms are incrementally added to a Ni (111) slab covered by graphene layer [17], a decrease of adhesion energy was observed with increasing carbon concentration within the Ni metal. In the case of SWCNT tangential growth where carbon concentration inside nanoparticle is low, the adhesion energy between $\mathrm{sp}^{2}$ carbon wall and catalyst nanoparticle is higher, thus prohibiting the dewetting of carbon nanotube wall on catalyst. As carbon wall dewetting is a necessary step for SWCNT nucleation and prolongation [20], the relatively strong adhesion energy favors the adhesion and wetting of the carbon $\mathrm{sp}^{2}$ wall on the surface of the nanoparticle, causing the catalyst particle deactivation and subsequent carbon layer encapsulation with further addition of carbon atoms to the SWCNT open ends. A typical example of such encapsulation is shown in Fig. 5b, where a graphite layer encapsulation is clearly seen. Indeed, 
such kind of carbon layer encapsulation is also displayed in TEM images of Fig. 2a and Fig. 4a. In contrast, nanoparticles, catalyzing SWCNT growth by perpendicular mode, has a weak adhesion with tube wall and cannot be easily deactivated by carbon encapsulation, resulting in a longer catalyst lifetime and therefore long tubes (ESI Fig. S6). It is noted that although the morphology of catalyst particle could fluctuate before or during CVD growth because of factors like Ostwald ripening [24], subsurface diffusion inside substrate [25] and tube-particle interaction [20], SWCNTs could preserve their chiralities along their whole lengths. Whether SWCNTs with different growth modes have different growth rates is still an open question and further in situ studies, like environmental TEM experiments are required to clarify this issue.

Based on the above discussions, we can now revisit some papers and interpret their experimental results using growth mode. As raising the carbon content inside the catalyst promotes a perpendicular growth mode, approaches that could increase carbon fraction should favor small diameter SWCNTs, hence narrow chirality distributions. Using a carbon source, that yields a high carburization of the catalyst, like CO, has been reported to favor the growth of SWCNTs enriched with near-armchair and small diameter species [4, 26-32]. During CVD, Co, Ni or Fe are generally adopted as the catalytically active components. Fig. 6 shows the UV-vis-NIR absorption spectra of SWCNTs grown on different catalysts under the same growth conditions as in our previous work $[26,31,32]$. Predominant growth of small diameter $(6,5)$ SWCNTs are obtained on Fe-based catalyst at ambient reaction pressure [26]. The chirality distributions of SWCNTs grown on Co and Ni-based catalysts are generally much broader and the diameters are larger. Besides $(6,5)$ species, the products include relatively large fractions of $(7,5),(8,4)$ and $(7,6)$ species with larger diameters $[31,32]$. Such growth results can be explained by the carbon solubility differences in the 
metal nanoparticles: Fe nanoparticles possess the highest carbon solubilities, facilitating perpendicular mode growth with smallest aspect ratio of $R_{d}$. It is also understandable that the gradual incorporation of $\mathrm{Fe}$ into $\mathrm{Ni}$ nanoparticles, from monometallic $\mathrm{Ni}$ to $\mathrm{Ni}_{0.27} \mathrm{Fe}_{0.73}$ alloy, shifts the most abundant species from $0.90 \mathrm{~nm}$-corresponding to a $(9,4)$ tube- for $\mathrm{Ni}$, to $0.82 \mathrm{~nm}-(7,5)$ tube for $\mathrm{Ni}_{0.27} \mathrm{Fe}_{0.73}$ alloy [30].

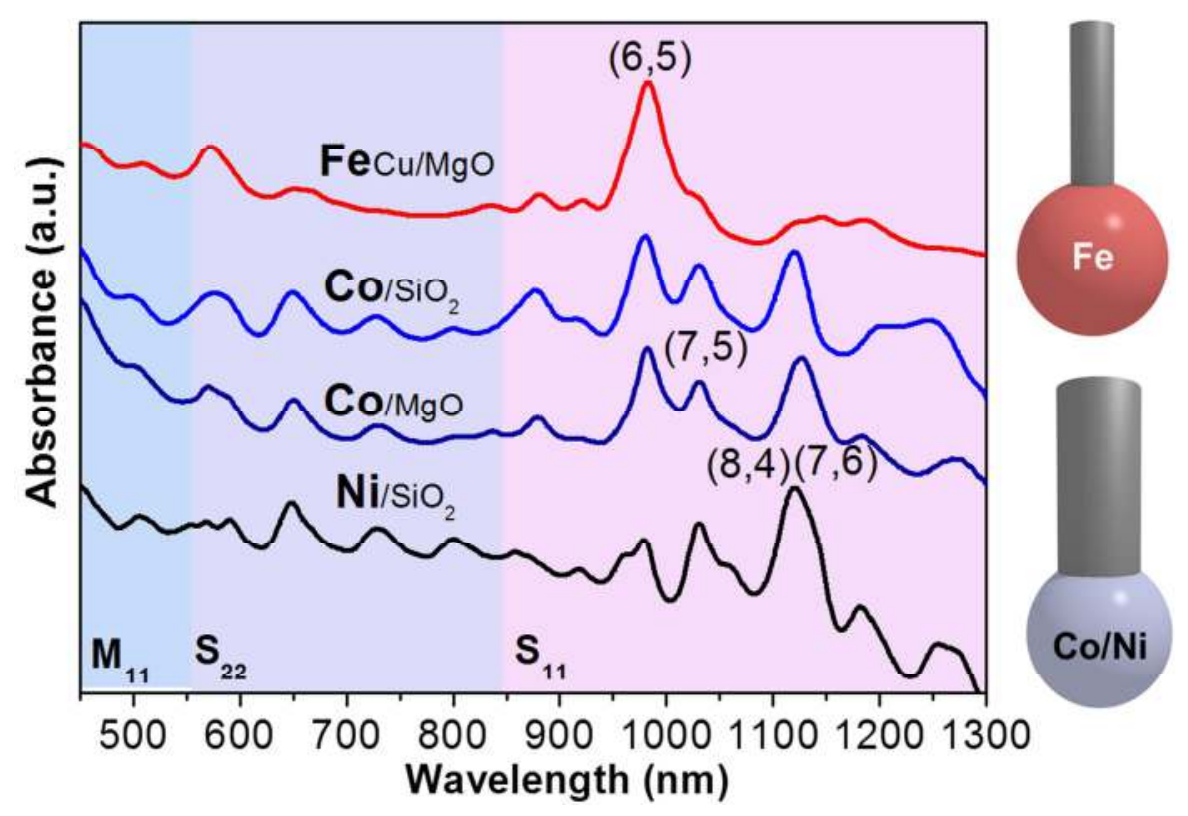

Figure 6. UV-vis-NIR adsorption spectra of SWCNTs grown on catalysts with different active components. All growth experiments are performed at $873 \mathrm{~K}$ using $\mathrm{CO}$ as the carbon precursor. Owing to the fact that high carbon solubility favoring perpendicular growth mode, SWCNTs grown on Fe nanoparticles [26] have much smaller diameters than those grown on Co [32] or Ni [21], as sketched in the schemes on the right.

\section{Conclusions}

To conclude, on the basis of a detailed TEM and ED analysis of a statistical number of SWCNTs, we unambigously show that there is no correlation between SWCNT lengths and their 
chiral angles. Instead, the tube length depends more on the diameter ratio between SWCNT and catalyst particle, i.e. the SWCNT growth mode. Atomistic computer simulations demonstrate that low carbon fractions in the catalyst lead to tangential growth, where the metallic nanoparticle wets the tube inner wall and can be easily encapsualted by the graphitic layers, facilitating the deactivation of the catalyst and the cessassion of SWCNT growth. On the contrary, catalyst nanoparticles with a high carbon fraction nucleate SWCNTs by perpendicular mode and can keep their activity for much longer time, accounting for the generation of long SWCNTs. In addition, since different growth modes correspond to different catalyst-tube contact regimes (line contact for perpendicular mode, and essentially surface contact in the tangential mode), they could lead to different ways of controlling the tube diameter and possibly structure. Our work thus provides a rational understanding of the key factor that determines SWCNT lengths and diameters, sheding more light on chirality-controlled synthesis of SWCNTs.

\section{Achknowledgement}

The research leading to these results has received funding from the European Union Seventh Framework Programme (FP7/2007-2013) under grant agreement $\mathrm{n}^{\circ} 604472$ (IRENA project) and French Research Funding Agency under grant No. ANR-13-BS10-0015-01 (SYNAPSE project). The authors would also like to acknowledge Scientific Research Foundation of Shandong University of Science and Technology for Recruited Talents (No. 2016RCJJ001), the Natural Science Foundation of China (No. 51272141) and Taishan Scholars Project of Shandong (No.

TS20110828). Dr. F. Ducastelle and Dr. F. Ding are acknowledged for helpful discussions. The 
work made use of the premises of Aalto University Nano-microscopy Center (Aalto-NMC) and Center for Scientific Computing (UCP).

\section{Supplementary data}

Extensive characterization results on nanoparticles and carbon nanotubes as described in the text.

\section{References}

[1] Yang F, Wang X, Zhang D, Qi K, Yang J, Xu Z, et al. Growing Zigzag $(16,0)$ Carbon Nanotubes with Structure-Defined Catalysts. J Am Chem Soc. 2015;137:8688-91.

[2] Yang F, Wang X, Zhang D, Yang J, Luo D, Xu Z, et al. Chirality-specific growth of single-walled carbon nanotubes on solid alloy catalysts. Nature. 2014;510:522-4.

[3] Zhao Q, Xu Z, Hu Y, Ding F, Zhang J. Chemical vapor deposition synthesis of near-zigzag single-walled carbon nanotubes with stable tube-catalyst interface. Science Advances. 2016;2:e1501729.

[4] Bachilo SM, Balzano L, Herrera JE, Pompeo F, Resasco DE, Weisman RB. Narrow (n,m)-distribution of single-walled carbon nanotubes grown using a solid supported catalyst. J Am Chem Soc. 2003;125:11186-7.

[5] He M, Jiang H, Liu B, Fedotov PV, Chernov AI, Obraztsova ED, et al. Chiral-selective growth of single-walled carbon nanotubes on lattice-mismatched epitaxial cobalt nanoparticles. Sci Rep. $2013 ; 3: 1460$.

[6] Sanchez-Valencia JR, Dienel T, Gröning O, Shorubalko I, Mueller A, Jansen M, et al. Controlled synthesis of single-chirality carbon nanotubes. Nature. 2014;512:61-4. 
[7] Ding F, Harutyunyan AR, Yakobson BI. From the Cover: Dislocation theory of chirality-controlled nanotube growth. Proc Natl Acad Sci. 2009;106(8):2506-9.

[8] Artyukhov VI, Penev ES, Yakobson BI. Why nanotubes grow chiral. Nature Commun. 2014;5.

[9] Yuan Q, Ding F. How a zigzag carbon nanotube grows. Angew Chem Int Ed. 2015;127(20):6022-6.

[10]Rao R, Liptak D, Cherukuri T, Yakobson BI, Maruyama B. In situ evidence for chirality-dependent growth rates of individual carbon nanotubes. Nat Mater. 2012;11:213-6.

[11]Inoue T, Hasegawa D, Chiashi S, Maruyama S. Chirality analysis of horizontally aligned single-walled carbon nanotubes: decoupling populations and lengths. Journal of Materials Chemistry A. 2015;3:15119-23.

[12] Dresselhaus MS, Dresselhaus G, Jorio A, Souza Filho AG, Pimenta MA, Saito R. Single Nanotube Raman Spectroscopy. Acc Chem Res. 2002;35:1070-8.

[13]Jorio A, Filho AGS, Dresselhaus G, Dresselhaus MS, Saito R, Hafner JH, et al. Joint density of electronic states for one isolated single-wall carbon nanotube studied by resonant Raman scattering. Phys Rev B. 2001;63:303-6.

[14]He M, Duan X, Wang X, Zhang J, Liu Z, Robinson C. Iron catalysts reactivation for efficient CVD growth of SWNT with base-growth mode on surface. J Phys Chem B. 2004;108:12665-8.

[15]Jiang H, Nasibulin A, Brown D, Kauppinen E. Unambiguous atomic structural determination of single-walled carbon nanotubes by electron diffraction. Carbon. 2007;45:662-7.

[16] Amara H, Roussel J-M, Bichara C, Gaspard J-P, Ducastelle F. Tight-binding potential for atomistic simulations of carbon interacting with transition metals: Application to the Ni-C system. Phys Rev B. 2009;79:014109. 
[17]Weatherup RS, Amara H, Blume R, Dlubak B, Bayer BC, Diarra M, et al. Interdependency of subsurface carbon distribution and graphene-catalyst interaction. $J$ Am Chem Soc. 2014;136:13698-708.

[18]Frenkel D, Smit B. Understanding Computer Simulation Academic Press, London. 1996.

[19]Fiawoo MFC, Bonnot AM, Amara H, Bichara C, Thibault-Penisson J, Loiseau A. Evidence of correlation between catalyst particles and the single-wall carbon nanotube diameter: A first step towards chirality control. Phys Rev Lett. 2012;108:195503.

[20]Diarra M, Zappelli A, Amara H, Ducastelle F, Bichara C. Importance of carbon solubility and wetting properties of nickel nanoparticles for single wall nanotube growth. Phys Rev Lett. 2012;109:185501.

[21]He M, Amara H, Jiang H, Hassinen J, Bichara C, Ras RH, et al. Key roles of carbon solubility in single-walled carbon nanotube nucleation and growth. Nanoscale. 2015;7:20284-9.

[22] Magnin Y, Zappelli A, Amara H, Ducastelle F, Bichara C. Size dependent phase diagrams of nickel-carbon nanoparticles. Phys Rev Lett. 2015;115:205502.

[23] Naidich YV, Perevertailo V, Nevodnik G. Wetting of graphite by nickel as affected by the liquid-phase dissolution process of carbon. Powder Metall Met Ceram. 1971;10:45-7.

[24]Amama PB, Pint CL, McJilton L, Kim SM, Stach EA, Murray PT, et al. Role of water in super growth of single-walled carbon nanotube carpets. Nano Lett. 2009;9:44-9.

[25] Kim SM, Pint CL, Amama PB, Zakharov DN, Hauge RH, Maruyama B, et al. Evolution in catalyst morphology leads to carbon nanotube growth termination. J Phys Chem Lett. 2010;1:918-22. 
[26] He M, Chernov AI, Fedotov PV, Obraztsova ED, Sainio J, Rikkinen E, et al. Predominant $(6,5)$ single-walled carbon nanotube growth on a copper-promoted iron catalyst. J Am Chem Soc. 2010;132:13994-6.

[27]Wang B, Poa CHP, Wei L, Li L-J, Yang Y, Chen Y. (n,m) Selectivity of Single-Walled Carbon Nanotubes by Different Carbon Precursors on Co-Mo Catalysts. J Am Chem Soc. 2007;129:9014-9.

[28] Wang H, Wang B, Quek X-Y, Wei L, Zhao J, Li L-J, et al. Selective synthesis of $(9,8)$ single walled carbon nanotubes on cobalt incorporated TUD-1 catalysts. J Am Chem Soc. 2010;132:16747-9.

[29]Zoican Loebick C, Podila R, Reppert J, Chudow J, Ren F, Haller GL, et al. Selective synthesis of subnanometer diameter semiconducting single-walled carbon nanotubes. J Am Chem Soc. 2010;132:11125-31.

[30]Chiang W-H, Mohan Sankaran R. Linking catalyst composition to chirality distributions of as-grown single-walled carbon nanotubes by tuning $\mathrm{Ni}_{\mathrm{x}} \mathrm{Fe}_{1-\mathrm{x}}$ nanoparticles. Nat Mater. 2009;8:882-6.

[31]He M, Chernov AI, Obraztsova ED, Sainio J, Rikkinen E, Jiang H, et al. Low temperature growth of SWNTs on a nickel catalyst by thermal chemical vapor deposition. Nano Res. $2011 ; 4: 334-42$.

[32]He M, Chernov AI, Fedotov PV, Obraztsova ED, Rikkinen E, Zhu Z, et al. Selective growth of SWNTs on partially reduced monometallic cobalt catalyst. Chem Commun. 2011;47:1219-21. 